\title{
Letters
}

\section{Diabetes in a population-based series of twin pairs discordant for leisure sedentariness}

Dear Sir,

Observational studies have suggested that physically active lifestyles decrease risk of Type II (non-insulin-dependent) diabetes mellitus [1,2]. Genetic selection could, however, explain some of this association. We analysed the incidence of Type II diabetes among same-sex twin pairs of known zygosity, who in 1975 were healthy and discordant for physical activity behaviour, selected from the Finnish Twin Cohort [3, 4].

The target group consisted of cohort subjects aged 25 to 64 years on 1 January 1976. Because chronic disease can restrict the ability to exercise, we excluded at baseline subjects with at least one of the following chronic diseases: diabetes, cardiovascular disease except for hypertension or venous diseases, chronic obstructive pulmonary diseases and subjects who had incident malignant cancer before 1977 [4]. These exclusions left 1637 monozygotic (MZ) and 3664 dizygotic (DZ) twin pairs healthy at baseline. Among these pairs there were 293 male and 328 female twin pairs discordant for leisure time physical activity behaviour at baseline (one twin reported not partaking in leisure physical activity at baseline in 1975 and at the same timepoint the co-twins leisure physical activity corresponded to at least one MET-hour/day [4]).

To further minimize the effect of occult antecedent disease, we allowed a lag period of at least one year after the physical activity assessment. We monitored incident cases of Type II diabetes from 1 January 1977 to the end of 1995 (19 years) based on three nationwide valid registers covering all Finnish citizens: the Hospital Discharge Register, Reimbursable Medications Register and Causes of Death Register [4].

According to these data, the 621 twin pairs discordant for leisure time physical activity at baseline included 20 twin pairs (5 MZ and $15 \mathrm{DZ}$ pairs; 15 male and 5 female pairs) among whom Type II diabetes occurred in one twin (18 of 20 had reimbursable medication which is only obtainable on production of a physician's certificate of illness). Among these 20 pairs discordant for incident Type II diabetes during follow-up 15 physically inactive and 5 physically active members contracted the

Corresponding author: U.M. Kujala, Unit for Sports and Exercise Medicine, University of Helsinki, Mannerheimintie 17, 00250 Helsinki, Finland disease (RR 3.00, 95\% CI 1.04-10.5 in the inactive twin compared with the active co-twin, $p=0.025$ by McNemar's test). In all 5 discordant $\mathrm{MZ}$ pairs the sedentary twin acquired Type II diabetes $(p=0.025)$. In addition, the physical activity discordant twin pairs included two pairs who were concordant for Type II diabetes at the end of our follow-up. Notably, the sedentary members of these twin pairs contracted Type II diabetes 7 years (MZ pair) and 8 years ( $D Z$ pair) earlier than the active co-twin.

Among the discordant MZ twin pairs mean body-mass index $\left(\mathrm{kg} / \mathrm{m}^{2}\right)$ at baseline tended to be higher among the sedentary member (26.9 SD 3.5) of the twin pair compared with the co-twin (25.9 SD 1.8). Concomitant differences in body mass index could be one explanatory mechanism but our study does not permit a cause and effect evaluation of associations between physical activity and body mass index.

Genetic effects on morbidity and mortality are under intensive scrutiny, with specific predisposing genes continually being identified. In the present context, genetic selection is claimed to explain much of the suspected health benefits of physical activity recorded in observational studies. Studying twins enabled us to adjust for genes and other familial factors. We found evidence that physical activity prevents diabetes despite gene predisposition, because active members of both dizygotic and monozygotic twin pairs had low incidence of Type II diabetes compared with their sedentary co-twins.

Yours Sincerely,

UM. Kujala, J. Kaprio, M. Koskenvuo

\section{References}

1. Manson JE, Rinun EB, Stampfer MJ et al. (1991) Physical activity and incidence of non-insulin-dependent diabetes in women. Lancet 338: 774-778

2. Ivy X (1997) Role of exercise training in the prevention and treatment of insulin resistance and non-insulin-dependent diabetes mellitus. Sports Med 24: 321-336

3. Kaprio J, Sarna S, Koskenvuo M, Rantasalo I (1978) The Finnish Twin Registry: Formation and compilation, questionnaire study, zygosity determination procedures and research program. Prog Clin Biol Res 24B:179-184

4. Kujala UM, Kaprio J, Sarna S, Koskenvuo M (1998) Relationship of leisure-time physical activity and mortality. The Finnish Twin Cohort. JAMA 279: 440-444 